\title{
Les multiples facettes de la fonction d'enseignant-ressource
}

\author{
Auteurs \\ Nathalie Deslauriers, Directrice école de l'Odyssée, Commission scolaire \\ des affluents, Canada, \\ nathalie.deslauriers@csda.ca
}

Maxime Coulombe-Godbout, Directeur-adjoint école de l'Odyssée, Commission scolaire des affluents, Canada,

maxime.coulombe-godbout@csda.ca 


\section{REVUE HYBRIDE DE L'ÉDUCATION}

\section{Résumé}

Dès l'année 2006, l'école secondaire de l'Odyssée a mis en place des enseignants-ressources pour fournir une aide individualisée aux élèves jugés comme étant à risque. Après avoir constaté les bénéfices de ce service et les difficultés des enseignants-ressources pour faire face à la demande, la direction a modifié l'approche individuelle pour une formule basée sur des groupes de besoins, puis a opté pour le coenseignement. Mis à l'essai dans les classes de français et de mathématiques, le dispositif de coenseignement a apporté une grande satisfaction aux enseignants impliqués et a donné des résultats inespérés avec les élèves en difficulté.

Mots-clés : enseignant-ressource, coenseignement, réussite, élèves HDAA, collaboration 


\section{$\&$}

\section{REVUE HYBRIDE DE L'ÉDUCATION}

\section{Mise en contexte}

L'intégration des élèves en situation de handicap ou en difficulté d'adaptation ou d'apprentissage (HDAA) au sein de la classe ordinaire au tournant des années 2000 a amené son lot de défis et de revendications de la part du personnel scolaire, particulièrement des enseignants. La convention collective nationale 2005-2010 de la FSE-CSQ allait ajouter une ressource consacrée à ces élèves et leurs enseignants par la mise en place du rôle d'enseignant-ressource. L'enseignant libéré d'une partie de sa tâche d'enseignement allait être appelé à soutenir les élèves HDAA, les enseignants et les autres intervenants de l'école.

L'école secondaire de l'Odyssée, école la plus défavorisée de la commission scolaire des Affluents (CSDA), a dès lors pu libérer un certain nombre d'enseignants dédiés à cette fonction, dont le mandat devait être peaufiné et adapté au milieu. À ses débuts, cette nouvelle ressource était utilisée pour le suivi individualisé des élèves catégorisés comme étant à risque, soit les doubleurs, les chevaucheurs ou les élèves provenant de la classe-ressource. Très rapidement, les enseignants-ressources de mathématiques et de français ont été appelés à entrer dans les classes en support à leurs collègues. En français, la présence de ce deuxième enseignant facilitait la mise en œuvre du « profil scripteur », pratique jugée gagnante dans la démarche d'écriture, alors qu'en mathématiques, les enseignants-ressources offraient un support ponctuel en fonction de la planification globale. Bien que les bénéfices de ce service offert entre 2006 et 2016 n'aient pas été mesurés, force est de constater que ses résultats ont été davantage perceptibles lorsque les interventions étaient dispensées en classe plutôt qu'en dehors, notamment au regard des résultats scolaires des élèves.

\section{Changement de paradigme}

En juin 2016, après avoir constaté la difficulté éprouvée par les enseignants-ressources offrant du suivi individualisé pour répondre aux besoins de tous les élèves jugés à risque, l'équipe de direction a voulu trouver un moyen de former des sous-groupes de besoin. Le "profil motivationnel » a été retenu, puis un questionnaire a été administré à tous les élèves du $1^{\mathrm{er}}$ cycle, ainsi qu'aux élèves doubleurs et chevaucheurs du $2^{\mathrm{e}}$ cycle, à la rentrée 2016. L'analyse de ces questionnaires devait permettre de regrouper les élèves à partir des 13 composantes de la motivation. Toutefois, cette entreprise s'est avérée fastidieuse et ne cernait pas clairement les méthodes d'enseignement pour soutenir l'apprentissage. La lecture du document "La fonction d'enseignantressource au secondaire » de Granger et Dubé (2014, 2016), suivi d'une discussion avec Nancy Granger, professeure à l'université de Sherbrooke, a permis de rectifier le tir et de proposer des interventions en classe pour soutenir les élèves et les aider à réussir. 


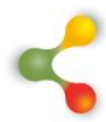

\section{REVUE HYBRIDE DE L'ÉDUCATION}

Dès lors, le défi a été lancé aux trois enseignants-ressources dont le précédent mandat était de faire du suivi individualisé, d'entrer dans les classes afin de vivre des expériences de coenseignement avec les enseignants volontaires, et ce, sans égard aux disciplines. Les autres enseignants-ressources " matière » poursuivaient leur mandat en allant en classe pour soutenir les élèves à différents moments jugés plus exigeants dans la planification de l'enseignement.

L'expérience vécue par une enseignante-ressource durant toute l'année scolaire nous a permis de constater que les stratégies d'enseignement efficace à mettre en place pouvaient différer en fonction du champ disciplinaire. En collaboration avec les enseignants-ressources, la direction a décidé de jumeler le français à l'univers social, et les mathématiques aux sciences, pour la prochaine année scolaire. Comme le choix des enseignants-ressources s'est effectué en fonction de ces deux champs disciplinaires, l'équipe s'est renouvelée.

\section{7-2018 : une année d'appropriation}

Dans les années antérieures s'est présenté le problème des horaires des enseignants ressources, notamment de leur complémentarité afin de couvrir les 36 périodes du cycle. La résolution de ce problème a été priorisée en prévision de la rentrée 2017 et les enseignants des quinze groupes du $1^{\text {er }}$ cycle ont pu ainsi faire appel au service.

Dès le début de l'année scolaire, tous les enseignants ont participé à une formation sur le coenseignement offerte par Philippe Tremblay, professeur à l'Université Laval. L'intention de la direction était de démontrer les bénéfices de cette pratique pour ensuite la multiplier dans l'école. Devant la réaction positive des enseignants, la direction a permis les initiatives de jumelage, qui allaient dans le sens du coenseignement, et ce, au-delà des périodes d'enseignement-ressource disponibles.

L'appariement des enseignants qui allaient pratiquer le coenseignement n'a jamais été un enjeu pour la direction ou les enseignants eux-mêmes, puisqu'ils étaient tous conscients d'œuvrer à la réussite des élèves, sans égard aux relations interpersonnelles. Des appariements étant plus naturels que d'autres, certains aménagements pouvaient être mis en place. À tire d'exemple, certains enseignantsressources préféraient retirer un petit groupe d'élèves ciblés pour travailler avec les notions vues en classe, mais dans un autre local. Cet enseignement parallèle permettait de voir les élèves sans incommoder l'enseignant qui ne se sentait pas prêt pour coenseigner avec un collègue. Un calendrier de rencontres en présence des chercheurs a été élaboré afin de favoriser un partage de pratiques entre les enseignants-ressources de l'école, et leur permettre d'obtenir des réponses à leurs questions. L'objectif de ces rencontres n'ayant pas été clairement défini par la direction, cellesci se sont avérées plus déstabilisantes qu'aidantes pour les enseignants- 


\section{$\&$}

\section{REVUE HYBRIDE DE L'ÉDUCATION}

ressources. Le sentiment d'efficacité professionnel des enseignantsressources était élevé vis-à-vis des pratiques liées au coenseignement. Ils étaient notamment préoccupés par l'efficacité du coenseignement à soutenir réellement les élèves en difficultés. À cet égard, le suivi offert régulièrement par les chercheurs a été bénéfique à deux duos d'enseignants qui avaient accepté d'être observés et de recevoir de la rétroaction sur les activités de coenseignement réalisées.

Informée des démarches qui avaient lieu au sein de notre école secondaire et de l'engagement du personnel dans ce projet, la direction générale adjointe de la CSDA a approché l'équipe de direction pour la mise en place d'un projet-pilote basé sur le coenseignement. Ce projet allait permettre à deux groupes d'élèves de bénéficier du coenseignement de façon intensive en français et en mathématiques de $2^{\mathrm{e}}$ secondaire, durant l'année scolaire 2018-2019.

\section{Quelques résultats}

Le groupe de coenseignement intensif a été formé d'une part, d'élèves qui fréquentaient la classe-ressource en $1^{\text {re }}$ secondaire et, d'autre part, d'élèves tout-venant dont certains effleuraient la note de passage en mathématiques ou en français. Au total, 32 élèves composaient ce groupe. Les enseignantes de français et de mathématiques étaient assistées par un enseignant-ressource formé à la base en enseignement spécialisé. L'enseignant-ressource travaillait, à temps plein avec ces deux groupes disciplinaires (mathématiques et français). Afin de choisir son personnel, la direction avait demandé à l'enseignant-ressource de suggérer deux personnes avec lesquelles il avait des affinités pédagogiques. Les personnes ciblées ont été rencontrées, leur mandat a été établi et les binômes ont pu débuter dès le but de l'année scolaire.

\section{L'appropriation du dispositif}

$\mathrm{Au}$ terme de leur expérience, les enseignants impliqués dans ce projet ont mentionné être très fiers de l'expérience vécue. Ils ont dû apprendre à se connaître, s'adapter à la personnalité de chacun, proposer des activités collaboratives, accepter de vivre des incertitudes et oser des formules pédagogiques inhabituelles comme placer les élèves en îlots de travail et les faire verbaliser davantage. Ces expérimentations leur ont permis de voir les élèves différemment et de développer une meilleure acuité des difficultés vécues par ces derniers.

\section{La gestion de la classe}

Les échanges pédagogiques semblent avoir surtout porté sur la gestion de la classe, compte tenu du nombre élevé d'élèves qui la composait. Toutefois, l'espace restreint dont il disposait les a conduits à faire preuve de créativité. Différentes configurations de classe ont été 


\section{$\&$}

\section{REVUE HYBRIDE DE L'ÉDUCATION}

essayées si bien qu'ils souhaitaient introduire un aménagement flexible, l'an prochain, en vue de répondre aux besoins des élèves qui se sentent moins à l'aise dans un cadre traditionnel. Ils ont imaginé des tables hautes permettant de travailler debout, des bancs de sol et des coins favorisant la collaboration. Ils souhaitent aussi revoir l'utilisation d'outils technologiques de manière à soutenir davantage les élèves.

La collaboration établie entre les trois enseignants semble avoir agi comme facteur de protection. Un enseignant souligne se sentir plus motivé et être davantage engagé depuis qu'il travaille étroitement avec des collègues. Les trois enseignants verbalisent le plaisir qu'ils ont eu en classe, les interactions décontractées entre eux, mais aussi avec les élèves qui ont influencé le climat de classe et contribué à faire de la classe une petite communauté d'apprentissage.

\section{La réussite des élèves}

Des réussites inespérées ont eu lieu. En effet, le suivi soutenu semble avoir bénéficié aux élèves en difficulté. Des élèves pour qui les programmes axés sur l'emploi étaient la seule issue envisagée au départ de l'année scolaire ont réussi avec succès les cours des deux disciplines leur permettant de continuer leur cheminement en classe ordinaire. Forts de ce constat, les élèves et leurs enseignants sont motivés à poursuivre.

\section{Conclusion}

Le dispositif de coenseignement a rallié les enseignants autour de pratiques pédagogiques différenciées. La concertation entre les enseignants, leur souci de cohésion et leur appropriation de nouvelles formules pédagogiques ont eu un effet appréciable sur la réussite des élèves, mais aussi sur leur persévérance scolaire. II reste encore du chemin à parcourir, notamment en termes de pédagogie active et de planification didactique. Les enseignants témoignent de leur engagement et de la valeur ajoutée du coenseignement.

L'an prochain, ces enseignants poursuivront et d'autres démarreront une expérience similaire en $1^{\text {re }}$ secondaire. En tant que direction d'établissement, notre rôle sera de soutenir et d'appuyer les initiatives proposées, de générer des lieux de discussion et de valoriser la collaboration au sein de notre établissement. 


\section{REVUE HYBRIDE DE L'ÉDUCATION}

\section{Références}

Comité patronal de négociation pour les commissions scolaires francophones. (2007). Convention collective du personnel enseignant 2005-2010. CSQ. file:///C:/Users/cathe/Downloads/FSE Convention a ce jour.pd f

Granger, N. et Dubé, F. (2014). La fonction d'enseignant-ressource au secondaire pour optimiser la réussite des élèves à risque ou HDAA. Dans S. Briquet-Duhazé, C. Ouellet et N. Lavoie (dir.), Progrès en lecture-écriture chez des élèves du primaire au postsecondaire (p. 101-120). Harmattan.

Granger, N. et Dubé, F. (2016). La fonction d'enseignant-ressource au secondaire. Pour optimiser la réussite des élèves à risque ou HDAA. Montréal : Université du Québec à Montréal. Repéré à http://www.adel.uqam.ca/sites/default/files/BROCHURE\%20EN SEIGNANT\%20RESSOURCE 2016.pdf 